\title{
Parameter Amendment and Simulation on Spinning Force Thamasett Algorithm for Steel Cylinder Shape Parts
}

\author{
Yu Yang \\ Changchun University of Science and Technology,China \\ yangyu1983@sina.com
}

Key words:Parameters Amendement; Power Spinning; Simulation

\begin{abstract}
This thesis is focused on the spinning force acquired by electrical measuring method through a large number of spinning process experiment on cylinder workpieces of different materials. Comparing with the result gotten by Thamasett, the law of deviation on the calculation of spinning force on radial, axial and tangential cylinder is found and parameters are amended so that the large error caused by ignoring metal deposition before spinning rollers is overcome. Based on the revised algorithm,the simulation result of spinning force is in accordance with the one in electrical measuring method. This simulation experiment is available for the efficient algorithm on spinning force of cylinder workpieces, the setting and optimization of spinning process parameters. It saves a lot of time and cost needed by process experiment and the error of spinning force is found within $8 \%$,which is more accurate than $15 \%$ by Thamasette.
\end{abstract}

\section{Introduction}

Spinning is a kind of chipless extrusion forming technology. The molding quality of the spinning products is mainly affected by the parameters of spinning process; the main basis for the setting of spinning process parameters is the amount of spinning force applied to the spinning rollers in the process of spinning. At present, the Thamasett Algorithm is generally used to calculate the spinning forces applied to the spinning rollers[1-2]. This algorithm is adopted under the assumed condition that the metals are flowed axially in a stable and continuous manner; however, during actual spinning, the metal buildup caused due to the unsmooth flowing of metal will change the deformation rate of the spinning products and this is also the primary cause[3] for the errors of being over $15 \%$ existed in the values of spinning force obtained respectively by this algorithm and the Electrometric Method in actual process experiment. Just because of the inaccuracy of the calculated values, the spinning process parameters are more inclined to be set up in the process of spinning by the experienced researchers on the basis of the calculated values; after being set up, the process parameters are still required to be modified by several times of process experiments; and the required machining quality and production efficiency are hard be fulfilled[4].

This paper, based on a large number of process experiments, discovers the deviation laws of the two values as follows in aspects of radial, axial and tangential spinning forces: the calculated value obtained by Thamasett Algorithm and the actual value measured by the Electrometric Method in the process experiment; besides, the parameters of the algorithm have been modified based on the law. The result of spinning force calculated by the Thamasett Algorithm and that by the modified algorithm are utilized to set up the spinning process parameters respectively; the results of spinning process experiment show that the dimensional precision of diameter of products spun according to the modified algorithm is obviously superior to that spun according to the Thamasett Algorithm; however, the surface roughness shows no obvious improvement. This paper has, based on the modified algorithm, utilized the software of hypermesh and ansys to build models and conducted simulation experiments on the barrel shaped parts; the result regarding the spinning force obtained in the simulation experiments is basically consistent with that measured by the Electrometric Method in the process experiment. The simulation model can be applied in the rapid calculation of spinning force exerted on the spinning rollers in the process of spinning of barrel shaped parts and in the rapid setup and optimization of spinning process parameters. In addition, it can effectively shorten the time required to set up the spinning process parameters and save the cost for the spinning process experiment. The error in spinning force of spinning rollers obtained by this simulation model can be controlled within $8 \%$ and evident improvement is made compared with the 
largest error of being over $15 \%$ in spinning force obtained by the Thamasett Algorithm.

\section{Thamasett Algorithm}

Basic assumptions made in the Thamasett Algorithm:

The biconical spinning roller with a fillet radius of $r_{p}=0$ is adopted;

The gross spinning force $P$ is applied vertical to the contact surface of spinning roller and barrel shaped workblank; the contact force on the deformation zone can be ignored;

The contact surface can be approximately regarded as curved rectangle.

When the radial force $P_{r}$ is calculated, the following rolling force equation is adopted:

$P_{r}=\frac{b L_{d} \overline{\sigma_{m}}}{\eta}$

In this equation, $b$ represents length of horizontal projection of contact surface between spinning roller and barrel type of workblank;

$L_{d}$ represents the length of contact arc on the cross section of the contact surface between the spinning roller and barrel shaped workblank;

$\overline{\sigma_{m}}$ represents the average resistance to deformation of the material;

$\eta$ represents the deformation efficiency.

$b=\Delta \operatorname{tctg} \alpha_{\rho}$

In this equation, $\Delta t$ represents the reduction in thickness.

$L_{d}=\sqrt{R_{y} \Delta h}$

In this equation, $R_{y}$ represents the induction radius;

$\Delta h$ represents the reduction in wall thickness of spinning roller once the spinning roller turns a circle.

$R_{y}=\frac{2 R R_{\rho}}{R+R_{\rho}}$

In this equation, $R$ represents the radius of barrel shaped workblank;

$R_{\rho}$ represents the radius of spinning roller.

The radial force is expressed as follows:

$P_{r}=\frac{\Delta t \overline{\sigma_{m}} \cdot \sqrt{R_{y} f c t g \alpha_{\rho}}}{\eta}$

The radial force $P_{r}$ is vertical to the axial force $P_{z}$, then the axial force can be expressed as follows:

$$
P_{z}=\frac{\Delta \operatorname{tg} \alpha_{\rho} \overline{\sigma_{m}} \cdot \sqrt{R_{y} f \operatorname{ctg} \alpha_{\rho}}}{\eta}
$$

The tangential force $P_{i}$ can be expressed as follows:

$$
P_{i}=\frac{\Delta t f \overline{\sigma_{m}}}{\eta}
$$

\section{Spinning Process Experiment}

The spinning machine is mainly comprised of spindle box, spindle, mandrel, main lathe bed, side lathe bed, cross-shaped sliding table, spinning roller, tail jacking device, heating system, cooling system, hydraulic system and electric system, see Figure 1 for details. 


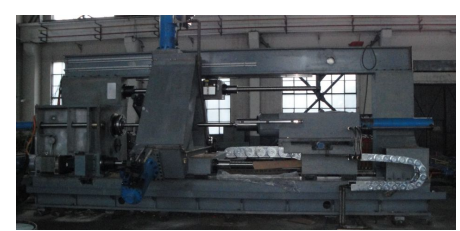

Figure1. Spinning machine

Working principle of metal spinning: mount and clamp the workblank on the mandrel and make it rotate together with the mandrel; meanwhile, the spinning roller is used to rotate around the workblank and relative feed is achieved between the spinning roller and mandrel in order to make the workblank to be pressed and produce continuous local plastic deformation; in this way, the hollow rotational parts can be obtained[5-11].

The spinning equipment adopted in the process experiment utilizes the barrel shaped parts of different materials to carry out the spinning process experiment and the experiment results are shown in the Table 1 as follows.

Table1 Experimental results

\begin{tabular}{|c|c|c|c|c|c|c|}
\hline \multirow{2}{*}{ Material } & \multicolumn{3}{|c|}{ Measured value(N) } & \multicolumn{3}{|c|}{ Theory value(N) } \\
\cline { 2 - 7 } & $\begin{array}{c}\text { Axial } \\
\text { force }\end{array}$ & $\begin{array}{c}\text { Radial } \\
\text { force }\end{array}$ & $\begin{array}{c}\text { Tangential } \\
\text { force }\end{array}$ & $\begin{array}{c}\text { Axial } \\
\text { force }\end{array}$ & $\begin{array}{c}\text { Radial } \\
\text { force }\end{array}$ & $\begin{array}{c}\text { Tangential } \\
\text { force }\end{array}$ \\
\hline StVIIu & 902 & 1127 & 129 & 1529 & 3273 & 120 \\
\hline StVIIlu & 1470 & 1519 & 137 & 1578 & 3381 & 111 \\
\hline StVIIlu & 2362 & 2166 & 211 & 2479 & 5321 & 137 \\
\hline StVIIu & 1470 & 1519 & 137 & 1578 & 3381 & 111 \\
\hline StVIIu & 1784 & 1950 & 210 & 1705 & 3646 & 170 \\
\hline
\end{tabular}

\section{Modification of Parameters}

It can be discovered from the comparison made between the spinning force of spinning rollers measured by Electrometric Method and that calculated by the Thamasett Algorithm that the deviation between the spinning force calculated by the Thamasett Algorithm and that actually measured is about $15 \%$ radially, $25 \%$ axially and $200 \%$ tangentially. According to this law, the modification of parameters is conducted on the Thamasett. Then the radial force applied to the spinning roller is expressed as follows:

$$
P_{r}=\frac{\Delta t \overline{\sigma_{m}} \cdot \sqrt{R_{y} f_{c t g} \alpha_{\rho}}}{\sqrt{2} \cdot 2.5 \eta}
$$

The axial force applied to the spinning roller is expressed as follows:

$$
P_{z}=\frac{\Delta \operatorname{tg} \alpha_{\rho} \overline{\sigma_{m}} \cdot \sqrt{R_{y} f \operatorname{ctg} \alpha_{\rho}}}{\eta}
$$

The tangential force applied to the spinning roller is expressed as follows:

$$
P_{i}=\frac{5 \Delta t f \overline{\sigma_{m}}}{4 \eta}
$$

\section{Effect of Spinning Force on the Molding Quality of Spinning Products}

The accuracy of spinning force calculated will have a direct effect on the setting of spinning process parameters, in which the feed amount will be most obviously influenced: excessive spinning forces will cause the increase in feed amount of spinning roller and then make the surface of barrel shaped part become corrugated; and the insufficient spinning forces will cause the decrease in feed amount and facilitate the failure of profiling of barrel shaped part. The effect of spinning force on the molding quality of spinning products is mainly manifested in two aspects: dimensional precision of diameter and surface roughness. Select the barrel shaped part used in Table 1 as the object of process experiment; use the values of spinning force of spinning rollers calculated by Thamasett Algorithm and modified algorithm to set up the process parameters; and carry out the spinning process experiments on the each group of barrel shaped parts . The results of experiment 
are shown in Table 2.

Table2 Experimental results

\begin{tabular}{|c|c|c|}
\hline & Size precision & harshness \\
\hline \multirow{4}{*}{ Correction algorithm } & $0.301 \mathrm{~mm}$ & 6.3 \\
\cline { 2 - 3 } & $0.300 \mathrm{~mm}$ & 6.3 \\
\cline { 2 - 3 } & $0.305 \mathrm{~mm}$ & 6.3 \\
\cline { 2 - 3 } & $0.303 \mathrm{~mm}$ & 6.3 \\
\cline { 2 - 3 } & $0.302 \mathrm{~mm}$ & 6.3 \\
\hline \multirow{4}{*}{$\begin{array}{c}\text { Thamasett } \\
\text { algorithm }\end{array}$} & $0.320 \mathrm{~mm}$ & 6.3 \\
\cline { 2 - 3 } & $0.314 \mathrm{~mm}$ & 6.3 \\
\cline { 2 - 3 } & $0.315 \mathrm{~mm}$ & 6.3 \\
\cline { 2 - 3 } & $0.317 \mathrm{~mm}$ & 6.3 \\
\hline
\end{tabular}

It is known from the results of experiment that the modified algorithm can obviously improve the accuracy of setting of process parameters and also enhance greatly the surface molding quality of the spinning products, as shown in Figure 2 and Figure 3 for details; the two figures are shot by the microscope with a magnification of 20 times and show the working surfaces of products spun according to the process parameters set up by the Thamasett Algorithm and the modified algorithm respectively. It can be discovered through comparison that the modification of algorithm does not evidently improve the surface roughness of the barrel shaped part.

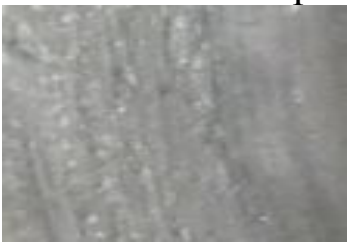

Figure 2. Taken with a microscope set algorithm based on Thamasett spinning process parameters from the surface of spinning products

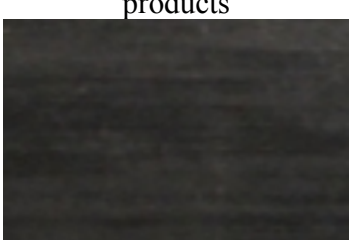

Figure 3. Taken with a microscope set according to the revised algorithm is spinning from the spinning process parameters the surface of products

\section{Simulation Experiment}

The modified algorithm is based to build a model to carry out the simulation experiment on spinning process. Firstly, the software CATIA is applied to build a geometric model, as shown inFigure 4.

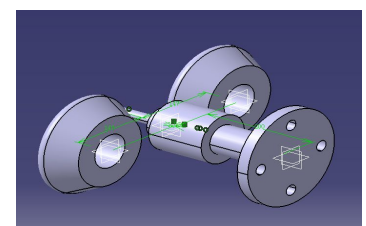

Figure 4. Geometry

The software Hypermesh is applied to conduct the pretreatment on the geometric model, as shown in Figure 5.

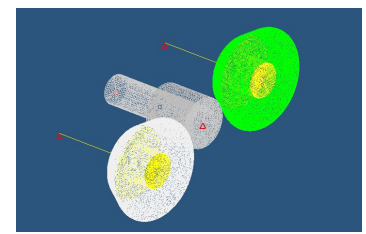

Figure 5. hypermesh pre-treatment

The software ansys is applied for calculation; the calculated spinning force of spinning roller in 
radial direction is shown in Figure 6; the spinning force in axial direction is shown in Figure 7; and the spinning force in tangential direction is shown in Figure 8.

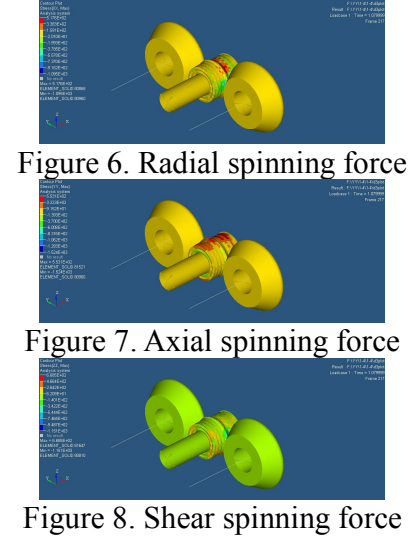

The spinning forces in radial, axial and tangential directions calculated in the simulation experiment are basically consistent with that measured by the Electrometric Method in the process experiment and its error produced is controlled within $8 \%$; as a result, greater improvement is achieved compared with the largest error of being over $15 \%$ calculated by the Thamasett algorithm.

\section{Conclusions}

This paper, based on a large number of process experiments, modifies the Thamasett Algorithm involved in the calculation of spinning force applied to the spinning rollers in the process of spinning made to the barrel shaped part; besides, the algorithms before and after being modified are based to set up the spinning process parameters and carry out the spinning process experiments; the results of the experiments show that the dimensional precision of diameter of the spinning products can be distinctly improved by applying the value of spinning force calculated according to the modified algorithm to set up the process parameter. Based on this algorithm, the software of hypermesh and ansys is used to build models and conduct simulation experiments of spinning process; the result regarding the spinning force obtained in the simulation experiments is basically consistent with that measured in the process experiment. The simulation model can be applied in the rapid calculation of spinning force in the process of spinning of barrel shaped parts and in the rapid setup and optimization of spinning process parameters. In addition, it can save lots of time and cost required to set up the spinning process parameters. The error in spinning force of spinning rollers obtained by this simulation model can be controlled within $8 \%$ and evident improvement is made compared with the largest error of being over $15 \%$ in spinning force obtained by the Thamasett Algorithm.

\section{References}

[1] Zhao Yunhao,Li Yanli.Spinning Technology and Application.Machinery Industry Press. 2008,5-6.

[2] Matsunok. Recent Research and Development in Metal Forming in Japan, J, Mater, Process, Technol,1997.6:1-3.

[3] Anon. Metal spinning in the auto motive industry, Sheet Metal Industries, 1995.12:13-18.

[4] Xue Kemin, Wang Zhen, Lu Yan. Elasto-plastic FEM Analysis and Experimental Study of Diametral Growth in Tube Spinning, J, Mater, Process, Technol,1997.69:172-175 .

[5] WANG Z T, XIE S S, JIN Q J. Elasto-plastic finite element analysis of hydrostatic extrusion with various mathematically contoured dies, Proceedings of the 24th International Machine Tool Design and Research Conference, eds, Davies BJ, Manchester,1983.09:51-58.

[6] HAYAMA, Hiroaki KUDO. Experimental Study of Tube Spinning. Bulletion of JSME. 1979.22:769-775. 
[7] Zhang Ning,Tan Wen,LiYonghua.Numerical Simulation and Spinning Force Analysis for Tube Stagger Spinning.Transactions of Shenyang Ligong University. 2009.28:55-58.

[8] Hu Lijin,Zhan Mei,Huang Liang.Prediction of radial crack of the workpiece during the splitting spinning based on ductile fracture criteria.Journal of Plasticity Enginnering. 2009.04:22-24.

[9] Li Zenghui,Wen Shubin,Han Dong.The Study of Spinning Precision Control for Thin Wall Cylinder with Large Length-diameter Ratio.China Metal Forming Equipment\&Manufacturing Technology. 2009.04:102-105.

[10] Mao Baiping,Shen Jian.Effects of Spinning Deformation on Microstructure and Properties of 6061 Al-alloy Tube.Hot Working Technology. 2008.37:49-54.

[11] Mu Shaozheng,Han Dong, Yang Yingli.Research on Tube Spinning Process and Propertied of Cast Titanium Alloy.China Metal Forming Equipment\&Manufacturing Technology. 2009.02:98-100. 